\title{
Effectiveness of the Intratissue Percutaneous Electrolysis (EPI®) technique and isoinertial eccentric exercise in the treatment of patellar tendinopathy at two years follow-up
}

\author{
Ferran Abat ${ }^{1}$ \\ Wayne-J Diesel ${ }^{2}$ \\ Pablo-E Gelber ${ }^{3}$ \\ Fernando Polidori ${ }^{1}$ \\ Joan-Carles Monllau ${ }^{3}$ \\ Jose-Manuel Sanchez-lbañez ${ }^{1}$ \\ 1 CEREDE, Sports Medicine, Barcelona, Spain \\ 2 Tottenham Hotspur FC, Hotspur Way, Enfield, Mid- \\ dlesex, UK \\ ${ }^{3}$ Department of Orthopedic Surgery, Hospital de la \\ Santa Creu i Sant Pau, Universitat Autònoma de \\ Barcelona, Barcelona, Spain. ICATME - Institut \\ Universitari Dexeus, Universitat Autònoma de \\ Barcelona, Barcelona, Spain
}

Corresponding author:

Ferran Abat

CEREDE, Sports Medicine, Barcelona

Dr. Roux 8-10, 08017 Barcelona, Spain

E-mail:drabat@cerede.es

\section{Summary}

Aim: to show the effect of Intratissue Percutaneous Electrolysis (EPI®) combined with eccentric programme in the treatment of patellar tendinopathy.

Methods: prospective study of 33 athlete-patients consecutively treated for insertional tendinopathy with Intratissue Percutaneous Electrolysis (EPIß) and followed for 2 years. Functional assessment was performed at the first visit, at three months and two years with the Tegner scale and VISA-P.

Results: an average improvement in the VISA-P of 35 points was obtained. The mean duration of treatment was 4.5 weeks. Some $78.8 \%$ of the patients returned to the same level of physical activity as before the injury by the end of treatment, reaching $100 \%$ at two years.

Conclusions: intratissue percutaneous electrolysis (EPI®) combined with an eccentric-based rehab program offers excellent results in terms of the clinical and functional improvement of the patellar tendon with low morbidity in a short-term period.

Level of Evidence: Therapy, level 4.
KEY WORDS: EPI, intratissue percutaneous electrolysis, patellar, tendinopathy, tenopathy, eccentric.

\section{Introduction}

The treatment of tendinopathy is a clinical challenge that some authors describe as one of the biggest problems in sports medicine ${ }^{1,2}$. Some studies suggest the use of the term tendinosis as it has proven to be more a degenerative condition rather than inflammatory $^{3}$. Furthermore, authors like Maffulli ${ }^{4,5}$ recommend using the term tendinopathy or tenopathy because it is a broader term that describes changes in and around the tendon. Recent studies emphasize the complex three-dimensional structure of the tendon ${ }^{6}$. Patellar tendon injuries are generally found on the insertional level at the attachment of the tendon at the inferior pole of the patella. It usually presents with pain in the tendon, tenderness to palpation and anterior knee pain ${ }^{1}$. Patellar tenopathy has a variable rate of prevalence and can reach 40 to $50 \%$ in sports like volleyball or those that involve jumping or braking ${ }^{1,2}$. The important degenerative changes in the development of tendinopathy are significant. They may even arrive at producing changes in the muscle ultrastructure after tendon rupture ${ }^{7}$.

Intratissue Percutaneous Electrolysis (EPI®) is an ultrasound-guided physiotherapeutic and medical technique that produces a non-thermal electrochemical ablation using a cathode flow directly oriented toward the tendon degeneration. The EPI $\AA^{\text {treatment causes }}$ an organic reaction that produces localized inflammation, exclusively in the treatment zone, that leads to rapid regeneration of injured tendon ${ }^{8-10}$.

Different techniques are currently used to treat patellar tenopathy ${ }^{11-13}$. The purpose of this paper is to show the effect of Intratissue percutaneous electrolysis $(E P I \circledast)$ guided by ultrasound together with an eccentric programme in the treatment of patellar tendinopathy. The working hypothesis is that EPI® combined with eccentric exercises improves the clinical aspect and functionality in patellar tendinopathy over a short period of time.

\section{Method}

It was a prospective study of 33 patients diagnosed with insertional patellar tendinopathy treated by the same therapist. The diagnosis of all patients was 
Effectiveness of the Intratissue Percutaneous Electrolysis (EPI囚) technique and isoinertial eccentric exercise in the treatment of patellar tendinopathy at two years follow-up

based on clinical examination and a color Doppler ultrasound study with a linear probe $(6-15 \mathrm{MHz})$.

The patients' demographic variables and pre-injury and post-treatment functional statuses were studied.

The clinical research ethics committee of our institution (08/062/0048) approved the study. To be included in the study, patients had to sign informed consent agreeing to treatment as well as the prospect of having pain in the lower insertional pole of the patella, living with the presence of pain for a minimum of 4 weeks, accepting the inability to continue participating in their sport and confirming an age of under 60 years old. Patients who presented with chronic arthropathy or another associated knee injury (such as a cruciate ligament injury or meniscopathy) were excluded. The use of anti-inflammatory drugs or corticosteroids was restricted throughout the first three months of the study. Patients received the Intratissue percutaneous electrolysis $(E P I \cap)$ technique treatment until there was clinical improvement or no improvement in the symptomology was seen after 10 sessions.

\section{Follow-up evaluation}

Functional assessment was performed using the validated scale of the Victorian Institute of Sport Assessment for the patellar tendon (VISA-P) ${ }^{14}$ and the Tegner scale. The VISA-P score ranges from 0 to a theoretical 100 when the patient is asymptomatic. The Tegner scale classifies patients according to their level of activity where zero is no activity or walking on a flat smooth floor and 10 is competitive sport at the highest level. The values of the scales were compiled from the written questionnaires given during patients' visits to the clinic; at the initial consultation, at discharge, at 3 months and in the evolutional control at 2 years. Patients were divided into two groups according to their initial symptomatology based on the
VISA-P score. Group 1 was made up of patients whose VISA-P value was less than 50 and Group 2 was those whose VISA-P value was greater than 50 . This division makes it possible to display the results depending on the degree of injury (more affected VISA-P $<50$ or less affected VISA-P $>50$ ).

At the same times as the functional assessment, patient satisfaction the EPI® treatment was evaluated with the Roles and Maudsley scale ${ }^{15}$. It classifies the degree of satisfaction as excellent (no pain and full activity), good (occasional discomfort with full activity), reasonable (occasional discomfort after prolonged activity) or poor (pain that limits activity).

\section{Treatment Protocol}

The EPI® technique described here should be performed with a specifically developed medically (EPI Advanced Medicine. Barcelona. Spain) certified device $^{9,10}$ (Directive 93/42/EEC) (Fig. 1A). It produces an adjustable galvanic current through a negative flow cathode electrode. For transmission of the flow to the treatment area, needles of from 0.30 to 0.32 $\mathrm{mm}$ in diameter and a modified electric scalpel are used (Fig. 1B). The intensity can be adjusted by changing both the duration and the milliamperes that are administered. Placement of the patient supine to minimize potential vagal reactions following the puncture is recommended. A thorough ultrasound inspection with a 6-15 MHz linear probe and color Doppler, following the European Society of Musculoskeletal Radiology guidelines ${ }^{16}$, was performed to permit the identification of any existent neovascularization (Fig. $2 A, B)$ and changes in terms of structural improvement and decreased neovascularization obtained with the EPI® treatment (Figs. 2 C,D). Preparation of the skin with isopropyl alcohol before puncture is required despite the bacteriostatic action the device has. Subsequently, 3 milliamps echo-guided punc-
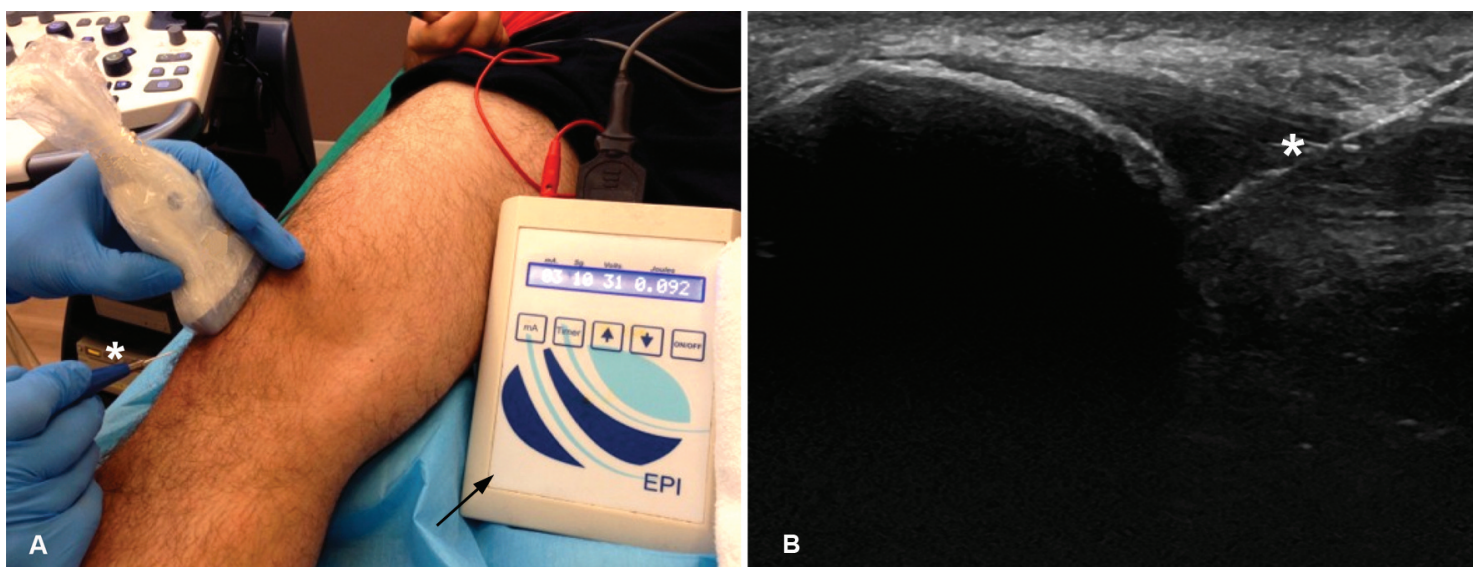

Figure 1 A-B. A. Device designed specifically to administer Intratissue percutaneous electrolysis (arrow). Echo-guided punctures $\left({ }^{*}\right)$ for the administration to specific areas of treatment with a $0.3 \mathrm{~mm}$ needle located with ultrasound targeting the treatment area. B. The image belongs to higher hyperechogenicity of the needle, increasing when the cathode flow passes form EPI® through it $\left(^{*}\right)$. 

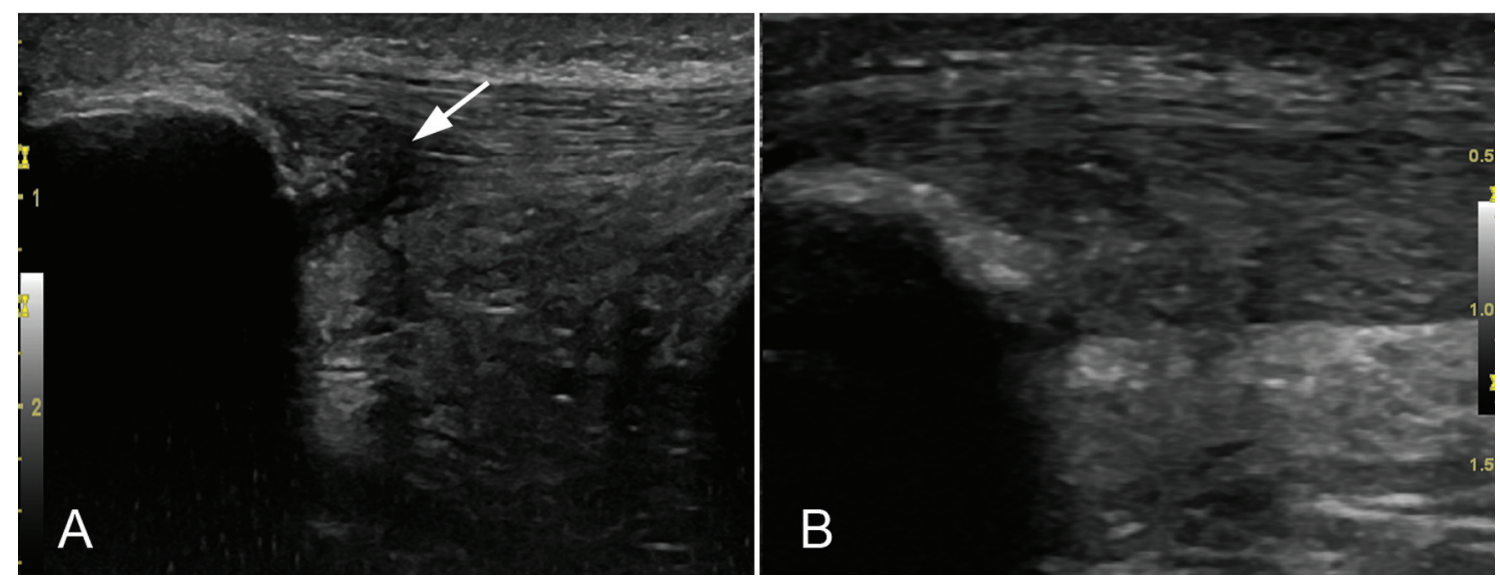

Figure $2 \mathrm{~A}-\mathrm{B}$. Ultrasound high-resolution Gray-scale longitudinal view with 6-15 $\mathrm{MHz}$ lineal probe image of the proximal patellar tendon pre-treatment with $\mathrm{EPI} B(\mathbf{A})$ and 3 months of treatment $(\mathbf{B})$ in the same patient. In the pre-treatment image (A) intensive hypoechoic zones (arrow) and thickened tendon is shown. At the lower pole of the patella, cortical irregularities were detected. In post EPI® treatment image (B) a significant decrease of the hypoechoic zones and echotexture improvement was detected (arrow).

tures are made with the device to obtain controlled debridement of the injured tendon. The debridement was assessed with the sonographic images.

All patients received a weekly session of $E P I \circledR$ and two weekly sessions of eccentric exercise using isoinertial resistance machines (YoYo ${ }^{\mathrm{TM}}$ Technology $\mathrm{AB}$, Stockholm, Sweden) consisting of 3 sets of 10 repetitions. Each repetition was performed with the concentric phase with both extremities whereas the eccentric phase was only performed with the affected limb at a maximum $60^{\circ}$ of knee flexion as recommended by Romero-Rodriguez ${ }^{17}$.

\section{Statistical Analysis}

Initially, the comparison of the basal situation of the study patients was taken up. Quantitative variables were described based on their mean value and standard deviation (SD). The comparison was terminated with a t-test of independent data, without assuming the existence of homoscedasticity. For ordinal variables, the median of each group as well as the minimum and maximum values were provided. For categorical variables, the percentage and the number of cases and inference using Fisher's exact test or Chisquare was calculated. An ANOVA study was used to analyze the different variables and the sphericity correction was carried out with the Greenhouse-Geisser test. Statistical analysis was performed with SPSS v.18 (SPSS Inc., Chicago, Illinois) with statistical significance set at 0.05 .

\section{Results}

Thirty-three patients were available for the final assessment at two years. Both groups were comparable in terms of in age $(p=0.536)$, gender $(p=0.335)$, domi- nant $\operatorname{limb}(p=0.398)$ or affected side $(p=0.093)$. The mean age was 25.3 years (range 16-53). The patellar tendon affectation was located in the dominant limb in $48.5 \%(n=16)$ of the patients. The patients consisted of some $12.1 \%(n=4)$ women and $87.9 \%(n=29)$ men. Some $57.6 \%(n=19)$ of the patients were football players, $3 \%(n=1)$ basketball players, $3 \%(n=1)$ played volleyball and the remaining $36.4 \%(n=12)$ engaged in other sports often involving vertical jumping. A sportsperson from first division or a similar classification by type of sport was considered professional. Second division sportspeople who were always paid for to practice it were considered semi-professional and amateurs were those who practiced sport with no economic incentive. Some $12.1 \%(n=4)$ were practicing their sport at the professional level, another $66.7 \%(n=22)$ at the semi-professional level and $21.2 \%(n=7)$ at the amateur level.

The mean duration of the symptoms of pain in the patellar tendon before coming to our center was 19 months (range 1-72 months). Patients had to have left their sport because of that pain for a mean period of 11.6 months (range $0-48$ ). Treatment with EPI ${ }^{\circ}$ lasted an average of 4.5 weeks (range 1-10) with a need for an average 4.4 sessions (range 1-10). According to the Roles and Maudsley scale, patient satisfaction at end of treatment at 3 months was excellent in 26 cases $(78.8 \%)$, good in 6 cases $(18.2 \%)$ and fair in 1 case (3\%). At two years follow up, $87.9 \%$ of the patients $(n=29)$ scored their satisfaction as excellent and the remaining $12.1 \%(n=4)$ as good. No adverse effects occurred during treatment or followup.

\section{Functional Results}

The overall pretreatment value of the VISA-P (Fig. 3) was $50.7 \pm 21.6$ points (range 10-90). This value in- 
Effectiveness of the Intratissue Percutaneous Electrolysis (EPI囚) technique and isoinertial eccentric exercise in the treatment of patellar tendinopathy at two years follow-up

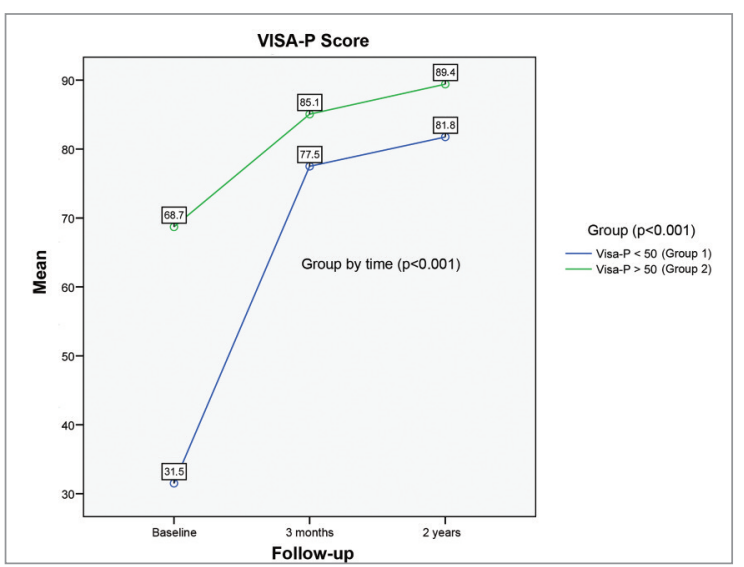

Figure 3. Column chart of the VISA-P values throughout follow up.

creased significantly to $81.4 \pm 12.8$ points (range 55 $100)$ at 3 months $(p<0.001)$ and maintained a slight improvement with $85.7 \pm 11.9$ points (range $60-100$ ) at 2 years $(p<0.001)$. Upon studying the value of the VISA-P groups, the fact that group 1 had a pretreatment value of $31.5 \pm 10.9$ points (range 10-48), improving to $77.5 \pm 15.3$ points (range 55-99) at 3 months $(p<0.001)$ and to $81.8 \pm 14.5$ points (range $60-99)$ at 2 years $(p<0.001)$ was observed. Similar behavior was seen in group 2 where the initial value of the VISA-P was $68.7 \pm 10.3$ points (range 52-90), improving to $85.1 \pm 9$ points (range $60-100$ ) at 3 months $(p<0.001)$ and to $89.4 \pm 7.6$ points (range 70 $100)$ at 2 years $(p<0.001)$. In the comparison between the two groups, it can be seen that at both 3 months $(p=0.091)$ and two years $(p=0.065)$, there are no differences in values on the VISA-P scale. The Greenhouse-Geisser test showed a statistical significance of $p<0.001$ for the different VISA-P values throughout the time intervals for the two groups. In turn, there were no statistically significant differences when comparing those patients who performed professional sports versus those who performed at the semi-professional or amateur level.

The average pre-injury Tegner value was 7.9 points (range 4-10), showing a value of 7.6 points (range 310) after three months of treatment, showing no statistically significant differences $(p=0.677)$ compared to the assessment at two years of 7.8 points (range 4-10). Group 1 (Fig. 4) started from a Tegner of 8 points (range 6-10) before treatment and reached 7.6 points (range 4-10) at 3 months and 7.9 points (range $5-10)$ at 2 years $(p=0.824)$. Group 2 showed a similar trend, starting from a pretreatment value of 7.8 (range 4-9), standing at 7.6 points (range $3-9$ ) at 3 months and 7.7 points (range 4.9) $(p=0.824)$ at two years. Of those patients who were considered professional athletes (pre-injury Tegner 10 points) at three months, $100 \%(n=4)$ returned to the same level of sport activity and stayed at the same level in the control at two years. Of the semi-professional group, with a pre-injury Tegner average of 8.1 (range $7-9$ ), $81.8 \%$

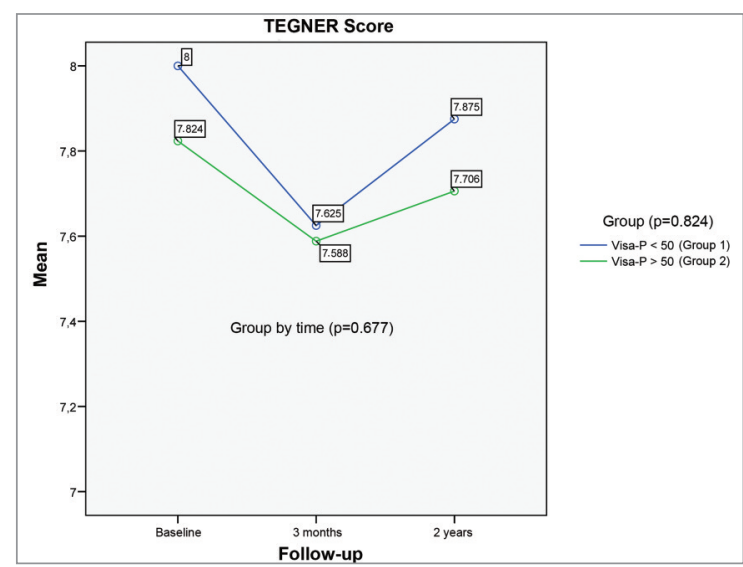

Figure 4. Lineal representation of the mean Tegner values during the follow-up.

$(n=18)$ were able to practice their sport at the same level as before the injury at 3 months and $18.2 \%$ $(n=4)$ were practicing at the control of two years. In the amateur group, which started from a pre-injury Tegner of 6 points (range 4-8), 57.1\% $(n=4)$ were able to return to their sport at the same level, while $42.9 \%(n=3)$ did it at the control at 2 years. Some $78.8 \%$ of the patients returned to the same level of physical activity as before the injury by the end of treatment, reaching $100 \%$ at two years. The relationship between treatment duration and Tegner values obtained are not significant $(p=0.677)$. The difference between the groups is not significant $(p=0.824)$.

\section{Discussion}

The principal finding of this study was the fact that when Intratissue Percutaneous Electrolysis (EPI®) was combined with eccentric exercise, superior results were found compared to studies using eccentrics only ${ }^{18-20}$. Equally good outcomes were achieved in a short period of time without comorbidities are shown in the presented treatment of insertional patellar tendinopathy.

The main limitation of this study is focused on combining intratissue percutaneous electrolysis (EPI®) with eccentric exercise. However, this combination is frequent in studies of tendinopathy. Thereupon, future studies should compare Eccentrics only versus Eccentrics plus EPIß. The lack of control group (difficult in private practice) and external validation must also be highlighted. Finally, a study with a mean follow-up time (two years) is presented. Thus, an RCT with a longer follow up would be necessary to demonstrate that this benefit lasts over time. Despite these limitations, this study provides the first analysis for up to two years of the treatment of patellar tendinopathy with intratissue percutaneous electrolysis (EPI®) in combination with eccentrics.

Percutaneous electro-stimulation with an electrolytic effect, denominated intratissue percutaneous electrol- 
ysis $(E P \mid \circledast)$, is a minimally invasive technique that involves the application of a galvanic current of high intensity through an acupuncture needle that stimulates a local inflammatory process in soft tissue. It makes phagocytosis and the repair of the affected tissue possible ${ }^{9,10}$. As shown in this work, electrolysis combined with eccentrics has brought about a notable improvement (average 35 point increase in the VISA-P) that allows for the resumption of sports activity to pre-injury levels in few sessions (mean 4.4), a short recovery period (average of 4.5 weeks) and low morbidity. Multiple therapies for the treatment of patellar tendinopathy have been put forward but not one of them has been set as the standard treatment ${ }^{18-30}$. The role of physiotherapy in the treatment of tenopathies remains unclear and it is not possible to draw any conclusions about its effectiveness based on scientific evidence ${ }^{19,20}$. Eccentric exercises are included within the few measures that have demonstrated efficacy in the treatment of these conditions. The problem is that the results are expressed in the medium and long term, between 3 to 6 months, with a mean cure of $40 \%$ to $60 \%$. Diathermy raises the temperature of the deep tissue from 41 to $45^{\circ} \mathrm{C}$ by means of electromagnetic energy. Recent research in long head biceps tendinopathy showed that hyperthermia is effective in the short-term, but it requires long-term monitoring to confirm its therapeutic effica$c y^{28}$. Extracorporeal shock wave is also used for sports physiotherapy and for the treatment of tenopathies. However, a meta-analysis performed by Maffulli et al. ${ }^{19}$ concluded that, on the basis of present knowledge, it is not recommended as a suitable protocol for the specific treatment of tenopathies.

In the case of the patellar tendon tenopathy, most patients opt for surgical treatment when conservative treatment fails. This achieves good or excellent results in $45 \%$ of the cases. These results are not higher than those obtained with eccentric exercise ${ }^{18}$. Recently, some novel methods have been proposed for the treatment of tenopathy. They include the likes of injections with platelet rich plasma $(P R P)^{27}$, injections with polidocanol ${ }^{26}$ and injections of aprotinin ${ }^{29}$. Then again, these techniques require further study to demonstrate their effectiveness and consistency in the medium or long term ${ }^{31}$.

\section{Conclusion}

The combination of Intratissue percutaneous electrolysis (EPIß) and eccentric exercise offers excellent results in terms of clinical and functional improvement in patellar tendinopathy with low morbidity in a half study period.

\section{Acknowledgments}

We are grateful to G. Gich for assisting in the statistical analysis. We also thank Eric Goode for his help in correcting the manuscript.

\section{References}

1. Zwerver J, Bredeweg SW, van den Akker-Scheek I. Prevalence of Jumper's knee among nonelite athletes from different sports: a cross-sectional survey. Am J Sports Med. 2011;39(9):1984-1988.

2. Renstrom PAHF, Woo SL-Y. Tendinopathy: a major medical problem in sport. In: Woo S, Renström P, Arnoczky S eds Tendinopathy in Athletes. Oxford, IK: Wiley-Blackwell 2008:1-9.

3. Soslowsky LJ, Thomopoulos S, Tun S et al. Overuse activity injures the supraspinatus tendon in an animal model: a histologic and biomechanical study. J Shoulder Elbow Surg. 2000;9(2):79-84.

4. van Dijk CN, van Sterkenburg MN, Wiegerinck JI, Karlsson J, Maffulli N. Terminology for Achilles tendon related disorders. Knee Surg Sports Traumatol Arthrosc. 2011;19(5):835-841.

5. Khan KM, Cook JL, Kannus P, Maffulli N, Bonar SF. Time to abandon the "tendinitis" myth. BMJ. 2002;324(7338):626-627.

6. Tresoldi I, Oliva F, Benvenuto M, et al. Tendon's ultrastructure. Muscles Ligaments Tendons J. 2013;3(1):2-6.

7. Zhang Q, Joshi SK, Manzano G, Lovett DH, Kim HT, Liu X. Original article Muscle extracellular matrix degradation and contractibility following tendon rupture and disuse. Muscles Ligaments Tendons J. 2013;3(1):35-41.

8. Abat F, Valles SL, Gelber PE, et al. Molecular repair mechanisms using the Intratissue Percutaneous Electrolysis technique in patellar tendonitis. Rev Esp Cir Ortop Traumatol. 2014;9. doi:10.1016/J.recot.2014.01.002

9. Sanchez-Ibañez JM. Clinical course in the treatment of chronic patellar tendinopathy through ultrasound guided percutaneous electrolysis intratissue $\left(\mathrm{EPI}()_{)}\right)$: study of a population series of cases in sport [PhD. Thesis]. Universidad de Leon. Leon. Spain 2013.

10. Sánchez- Sánchez JL. Comparative study of conventional physical therapy with one that includes Intratissue Percutaneous Electrolysis ${ }^{\circledR}$ technique in patients with chronic patellar tendinopathy [PhD. Thesis], Universidad de Salamanca. Salamanca. Spain. 2011.

11. Loppini M, Maffulli N. Conservative management of tendinopathy: an evidence-based approach. Muscles Ligaments Tendons J. 2012;1(4):134-137.

12. Andres BM, Murrell GA. Treatment of tendinopathy: what works, what does not, and what is on the horizon. Clin Orthop Relat Res. 2008;466(7):1539-1554.

13. Larsson ME, Käll I, Nilsson-Helander K. Treatment of patellar tendinopathy-a systematic review of randomized controlled trials. Knee Surg Sports Traumatol Arthrosc. 2012;20(8):16321646.

14. Hernandez-Sanchez S, Hidalgo MD, Gomez A. Cross-cultural adaptation of VISA-P score for patellar tendinopathy in Spanish population. J Orthop Sports Phys Ther. 2011;41(8):581591.

15. Roles NC, Maudsley RH. Radial tunnel syndrome: resistant tennis elbow as a nerve entrapment. J Bone Joint Surg Br. 1972;54(3):499-508.

16. Beggs I, Bianchi S, Bueno A, et al. ESSR Ultrasound Group Protocols. Musculoskeletal Ultrasound Technical Guidelines: Knee.

17. Romero-Rodriguez D, Gual G, Tesch PA. Efficacy of an inertial resistance training paradigm in the treatment of patellar tendinopathy in athletes: a case-series study. Phys Ther Sport. 2011;12(1):43-48.

18. Bahr R, Fossan B, Løken S, Engebretsen L. Surgical treatment compared with eccentric training for patellar tendinopathy (Jumper's Knee). A randomized, controlled trial. J Bone Joint Surg Am. 2006;88(8):1689-1698.

19. Rompe JD, Nafe B, Furia JP, Maffulli N. Eccentric loading, shock-wave treatment, or a wait-and-see policy for tendinopa- 
Effectiveness of the Intratissue Percutaneous Electrolysis (EPI囚) technique and isoinertial eccentric exercise in the treatment of patellar tendinopathy at two years follow-up

thy of the main body of tendon Achillis: a randomized controlled trial. Am J Sports Med. 2007;35(3):374-383.

20. Malliaras P, Barton CJ, Reeves ND, Langberg H. Achilles and patellar tendinopathy loading programmes: a systematic review comparing clinical outcomes and identifying potential mechanisms for effectiveness. Sports Med. 2013;43(4):267286.

21. Frizziero A, Fini M, Salamanna F, Veicsteinas A, Maffulli N, Marini M. Effect of training and sudden detraining on the patellar tendon and its enthesis in rats. BMC Musculoskelet Disord. 2011;12:20.

22. Alaseirlis DA, Konstantinidis GA, Malliaropoulos N, Nakou LS, Korompilias A, Maffulli N. Arthroscopic treatment of chronic patellar tendinopathy in high-level athletes. Muscles Ligaments Tendons J. 2013;2(4):267-272.

23. Pascarella A, Alam M, Pascarella F, Latte C, Di Salvatore MG, Maffulli N. Arthroscopic management of chronic patellar tendinopathy. Am J Sports Med. 2011;39(9):1975-1983.

24. Furia JP, Rompe JD, Cacchio A, Del Buono A, Maffulli N. A single application of low-energy radial extracorporeal shock wave therapy is effective for the management of chronic patellar tendinopathy. Knee Surg Sports Traumatol Arthrosc. 2013;21 (2):346-350.

25. Crisp T, Khan F, Padhiar N, et al. High volume ultrasound guided injections at the interface between the patellar ten- don and Hoffa's body are effective in chronic patellar tendinopathy: A pilot study. Disabil Rehabil. 2008;30(2022):1625-1634.

26. Willberg L, Sunding K, Forssblad M, Fahlström M, Alfredson $\mathrm{H}$. Sclerosing polidocanol injections or arthroscopic shaving to treat patellar tendinopathy/jumper's knee? A randomised controlled study. Br J Sports Med. 2011;45(5):411-415.

27. Yuan $\mathrm{T}$, Zhang $\mathrm{CQ}$, Wang $\mathrm{JH}$. Augmenting tendon and ligament repair with platelet-rich plasma (PRP). Muscles Ligaments Tendons J. 2013;3(3):139-149.

28. Oliva F, Via AG, Rossi S. Short-term effectiveness of bi-phase oscillatory waves versus hyperthermia for isolated long head biceps tendinopathy. Muscles Ligaments Tendons J. 2012;1(3):112-117.

29. Orchard J, Massey A, Brown R, Cardon-Dunbar A, Hofmann J. Successful management of tendinopathy with injections of the MMP-inhibitor aprotinin. Clin Orthop Relat Res. 2008;466(7):1625-1632.

30. Warden SJ, Metcalf BR, Kiss ZS, et al. Low-intensity pulsed ultrasound for chronic patellar tendinopathy: a randomized, double-blind, placebo-controlled trial. Rheumatology (Oxford). 2008;47(4):467-471.

31. Padulo J, Oliva F, Frizziero A, Maffulli N. Muscles, Ligaments and Tendons Journal. Basic principles and recommendations in clinical and field science research. MLTJ. 2013;4:250-252. 